\title{
ESTUDO COMPARATIVO ENTRE O XENODIAGNÓSTICO NATURAL E O ARTIFICIAL, EM CHAGÁSICOS CRÔNICOS
}

\author{
Adelair Helena dos Santos, Ionizete Garcia da Silva e Anis Rassi
}

\begin{abstract}
Foram realizados, simultaneamente, os xenodiagnósticos natural e artificial em 57 pacientes chagásicos, na fase crônica, com idades entre 26 e 83 anos, sendo 26 do sexo masculino e 31 do feminino, todos com sorologia reativa. Este estudo teve a finalidade de verificar a sensibilidade do método artificial, visando à sua utilização, em rotina de laboratório. Aplicaram-se 40 Dipetalogaster maximus de $1^{\circ}$ estádio para cada paciente tanto no xenodiagnóstico natural quanto no artificial. O xenodiagnóstico artificial for significativamente mais sensivel do que o natural para detectar o Trypanosoma cruzi, uma aplicaçao do artificial foi igual à do método natural aplicado três vezes. $A$ "xenopositividade" foi de 35,1\%, sendo significativamente maior no sexo masculino, nas faixas etârias de 56 a 65 anos e 66 a 83 anos. Estes resultados mostram a viabilidade e a possibilidade do uso do xenodiagnóstico artificial em rotina de laboratório.
\end{abstract}

Palauras-chaves: Xenodiagnóstico. Doença de Chagas. Trypanosoma cruzi. Fase crônica. Diagnóstico parasitológico.

O xenodiagnóstico tem sido utilizado para a comprovação parasitológica da doença de Chagas na fase crônica e tornou-se muito valioso pelos seguintes aspectos: econômico, de fácil aplicação, tanto em laboratório quanto no campo, útil no acompanhamento de testes com drogas tripanosomicidas, possibilitando avaliar o nível da parasitemia circulante $e$ isolar cepas $^{3}$. Em relação ao tratamento etiológico da doença de Chagas, o xenodiagnóstico tem sido um parâmetro importante na seleção de pacientes. Após o tratamento, a negatividade do xenodiagnóstico tem sido um parâmetro evidenciador da eficiência quimioterápica.

A associação dos fatores desfavoráveis ao xenodiagnóstico natural, com a necessidade de acompanhar tanto os pacientes chagásicos alérgicos à saliva do triatomíneo, como aqueles submetidos a tratamento com drogas imunossupressoras, ou transplantes de órgãos, que apresentam possibilidade de reagudização da doença, estimulou investigações sobre o

\footnotetext{
Laboratório de Biologia, Fisiologia de Insetos e Xenodiagnóstico do Instituto de Patologia Tropical e Saúde Pública, Universidade Federal de Goiás, Goiânia, GO. Suporte Financeiro: SENESU, FUNAPE, CONSTTEG. Endereço para correspondência: Prof. Ionizete Garcia da Silva. Laboratório de Biologia, Fisiologia de Insetos e Xenodiagnóstico/IPTSP/UFG. Caixa Postal 131, 74001-970 Goiânia, GO; Fax:(062) 2616414.

Recebido para publicação em 27/12/94.
}

xenodiagnóstico artificial, uma vez que os estudos sobre o assunto são raros e insuficientes para demonstrar a viabilidade de seu usO ${ }^{469141529}$.

Este trabalho teve a finalidade de verificar a possibilidade de se usar o xenodiagnóstico artificial em rotina de laboratório, tendo como objetivos a eliminação dos problemas de reação cutânea ${ }^{18}$ provocados pela saliva do triatomíneo, os casos de anafilaxia', e a possibilidade de fazer o exame em qualquer situação em que se encontre o paciente imunodeprimido, alérgico ou transplantado.

\section{MATERIAL E MÉTODOS}

Espécie de triatomíneo utilizada. Usouse Dipetalogaster maximus devido à recomendação de um simpósio e os resultados obtidos por vários estudos ${ }^{5} 132826$ mostraram ser essa espécie um bom meio de cultura in vivo para replicar o T. cruzi.

Para cada xenodiagnóstico aplicaram-se 40 ninfas de $D$. maximus, no primeiro estádio. Os triatomíneos foram criados em câmara climatizada $^{20}{ }^{22}$ e alimentados com sangue de $\operatorname{aves}^{2025}$. À medida que ocorria a oviposição, os ovos eram recolhidos e colocados em placas de Petri forradas com papel filtro, amarradas com elásticos, identificadas e colocadas para incubação a $28^{\circ} \mathrm{C}$. Após a eclosão, as ninfas eram contadas e transferidas para os frascos plásticos e mantidas na câmara biológica ${ }^{21}$, em 
jejum, por 15 dias, período necessário ao triatomíneo para atingir a repleção total ${ }^{27}$ (alimentaçào máxima, que geralmente ocorre num período de $10-15$ minutos). No entanto, com o propósito de padronizar o mesmo tempo para os dois experimentos, os triatomíneos ficaram expostos à alimentação por um período de 30 minutos, tanto no xenodiagnóstico natural como no artificial.

Caracterização morfológica da alimentação. Em qualquer um dos estádios, 0 $D$. maximus atinge a repleção total quando o seu abdome adquire a forma de um balào e o tegumento apresenta-se brilhante; desta forma, o triatomíneo completa o ciclo com apenas uma alimentação por estádio ${ }^{21}$. Foi observada esta característica para todos $D$. maximus de primeiro estádio; nessas condições, a ingestão média de sangue é cerca de $91,7 \mu 1^{2122}$.

Pacientes. Foram estudados 57 pacientes na fase crônica da doença de Chagas, todos com três provas sorológicas reativas (imunofluorescência indireta, hemaglutinação indireta e ELISA) e nenhum submetido a tratamento específico. O xenodiagnóstico foi aplicado no período matutino, entre 8 e $11 \mathrm{~h}$.

Xenodiagnóstico natural. Foram usados 10 frascos ${ }^{20} \mathrm{com}$ quatro triatomíneos em cada um, que foram acondicionados em pequenas sacolas de pano, amarradas na parte flexora do antebraço do paciente, ali permanecendo por aproximadamente 30 minutos $^{21}$. Após a alimentaçào, os frascos, com os triatomíneos, foram colocados na estufa incubadora a $28^{\circ} \mathrm{C}$. O xenodiagnóstico natural foi repetido por três vezes em cada paciente.

Xenodiagnóstico artificial. O sangue foi coletado através de punção venosa no antebraço, na veia cubital, usando-se tubos a vácuo, com capacidade de aspirar cerca de $7 \mathrm{ml}$ de sangue. Cada tubo tinha sido heparinizado com 143 unidades (Liquemine), o que corresponde à dose de 4 il de hepatina para $1 \mathrm{ml}$ de sangue. A quantidade de heparina do tubo foi considerada favorável à experimentação, visto não ocorrer coagulação sanguínea, nem a morte dos triatomíneos. Optou-se pela heparina, porque este anticoagulante apresentou menor interferência no desenvolvimento do triatomíneo e na replicação do $T$. cruzi $i^{11}$.

Para alimentaçào dos triatomíneos foi utilizado um dispositivo ${ }^{24}$ constituído de uma estante de madeira, uma fonte de aquecimento e uma peça de vidro (mamadeira) ${ }^{10}$ composta de duas câmaras: uma interna, em forma de funil, guarnecida ao fundo por uma membrana de látex (preservativo masculino não lubrificado), distendida de forma a aparecer o branco translúcido, e presa ao fundo $\mathrm{com}$ fita adesiva; e outra externa, que serviu para aquecer a primeira através de um fluxo contínuo de água a $37^{\circ} \mathrm{C}$, bombeada de um banho-maria (Figura 1).

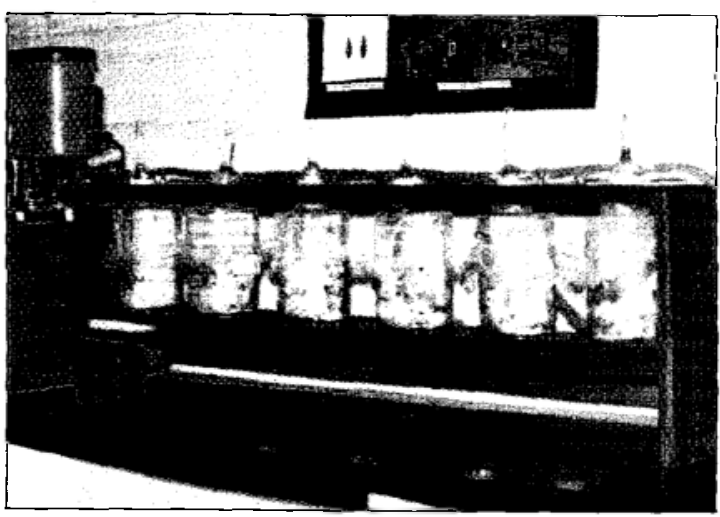

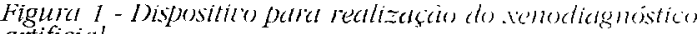
artificial.

O sangue colhido foi transferido para a "mamadeira" usando-se seringas descartáveis, com capacidade para 10ml, colocandorse o bisel da agulha, na parede do funil, e o êmbolo era comprimido lentamente, para evitar a formação de coágulos e espuma, fazendo com que o sangue descesse suavemente pela parede de vidro até a membrana de látex. Em seguida, colocou-se em contato com a membrana de látex, o frasco com os triatomíneos, para que esses se alimentassem, tendo-se o cuidado de se manter a temperatura constante, por um período de 30 minutos. Após a alimentação, os tubos foram guardados na câmara climatizada até serem realizadas as leituras.

Em ambos os métodos, as leituras foram realizadas aos 30, 60 e 90 dias após a aplicação do xenodiagnóstico, pelo método das dejeçôes espontâneas ${ }^{23}$.

Contagem de iripanosomas. Com o auxílio de um micropipetador e com a tampa aberta apenas o necessário para introduzi-lo no frasco, colhiam-se fezes ou urina, examinandoas entre lâmina e lamínula ao microscópio com aumento médio $(400 \mathrm{x})$ e contava-se o número de tripanosomas ${ }^{25}$.6. 
Análise estatística. Foram usados os testes do Qui-quadrado e de Wilcoxon, ambos ao nível de significância de 5\%.

\section{RESULTADOS}

Aplicaram-se, simultaneamente, o xenodiagnóstico natural e o artificial, em 57 pacientes chagásicos na fase crônica, para verificar a sensibilidade de cada um dos métodos. Desses pacientes, 10 tinham idades entre 26 e 39 anos; 22 entre 40 e 60 anos, e 25 com idades entre 61 e 83 anos. Verificou-se que o método artificial foi significativamente mais eficiente do que o método natural, para diagnosticar a doença de Chagas, pelo teste de Wilcoxon, com $\mathrm{p}<0,05$ (Tabela 1). Constatouse, também, que a positividade no método artificial $(28,1 \%)$ foi estatisticamente igual à do método natural aplicado três vezes $(29,8 \%)$, pelo teste do Qui-quadrado, ao nível de 5\%. A "xenopositividade" acumulada nos dois métodos e repetiçôes foi de 35,1\%. A aplicação seriada do xenodiagnóstico natural por três vezes, com intervalos de 30 dias, aumentou o percentual de positividade, mas não foi capaz de detectar o $T$. cruzi em dois dos vinte pacientes xenopositivos que foram positivos apenas pelo exame artificial (realizado uma vez).

O xenodiagnóstico foi aplicado em pacientes chagásicos adultos, com idades entre 26 e 83 anos. Verificou-se que a positividade do xenodiagnóstico foi significativamente maior nas faixas etárias de 56-65 e 66-83 anos, sendo maior no sexo masculino, pelo teste do $\mathrm{X}^{2}$, ao nivel de $5 \%\left(\mathrm{X}^{2}=13,73\right)$. A freqüência de positividade, de acordo com a faixa etária, está expressa na Figura 2, evidenciando-se a faixa etária de 41 a 55 como a mais difícil de se detectar o tripanosoma circulante.

Pelo estudo comparativo entre o número médio de tripanosomas excretados, no

Tabela 7 - Comparaçāo da positividade entre o xenodiagnóstico natural e o artificial, aplicado simultaneamente em 57 pacientes chagasicos na fase crônica.

\begin{tabular}{|c|c|c|c|c|c|c|c|}
\hline \multirow[t]{2}{*}{ Xenodiałgnóstico } & \multirow[t]{2}{*}{ No de pacientes } & \multicolumn{6}{|c|}{ Leituras/positividade } \\
\hline & & $1^{\mathrm{a}}$ & $\%$ & $2^{2}$ & $\%$ & $3^{2}$ & $\%$ \\
\hline Artificial & 57 & 10 & 17,5 & $\overline{16}$ & 28,1 & 16 & 28,1 \\
\hline Natural & 57 & 9 & 15,8 & 12 & 21,1 & 12 & 21,1 \\
\hline Natural $1^{2}$ repetição & 47 & 7 & 14,9 & 11 & 23,4 & 11 & 23,4 \\
\hline Natural $2^{2}$ repetiçào & 47 & 13 & 27,7 & 14 & 29,8 & 14 & 29,8 \\
\hline$N^{o}$ de examinados & 57 & 15 & $26, \overline{3}$ & 20 & $35, \overline{1}$ & 20 & 35,1 \\
\hline
\end{tabular}

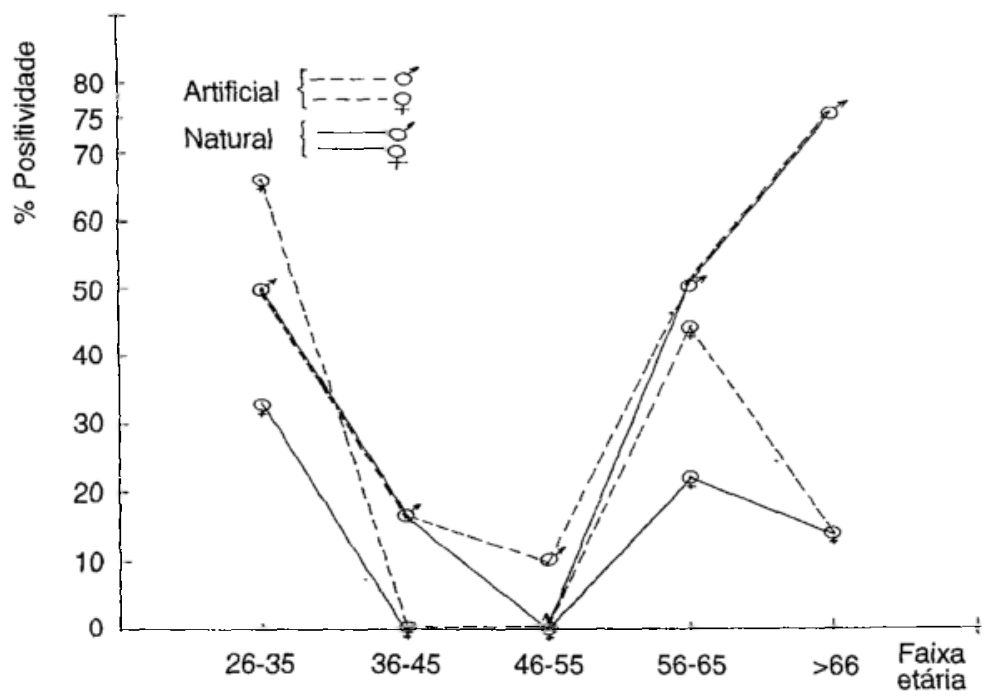

Figura 2 - Freqüência da positividade do xenodiagnóstico natural e artificial, de acordo com o sexo e a faixa etária, em cbagásicos na fase crônica. 
Santos AH, Silva IG, Rassi A. Estudo comparativo entre o xenodiagnóstico natural e o artificial em chagásicos crônicos. Revista da Sociedade Brasileira de Medicina Tropical 28:367-373, out-dez, 1995.

xenodiagnóstico artificial e no natural, verificou-se que a quantidade de tripanosomas foi significativamente maior no método artificial do que no natural (Tabela 3). Constatou-se, também, que houve um número significativamente maior de triatomíneos infectados pelo $T$. cruzi no método artificial (Tabela 2).

Verificoul-se que não havia necessidade de se realizar a terceira leitura (Tabela 1 ), pois a positividade do xenodiagnóstico fora a mesma na segunda (aos 60 dias).

\section{DISCUSSÃO}

Não foi encontrado na literatura pertinente, nenhum trabalho sobre o xenodiagnóstico artificial aplicado em pacientes chagásicos que possibilitasse uma efetiva comparação.

Com relação aos artifícios usados para a realização do xenodiagnóstico in vitro, vários estudos foram feitos sobre membranas para alimentação artificial de triatomíneos, que apontam a tripa de boi, como a membrana mais favorável do que a de plástico ou de

Tabela 2 - Indice de infecçâo de Dipetalogaster maximus pelo Trypanosoma cruzi. após aplicação simultânea do xenodiagnósico natural e artificial, em 57 pacientes chagásicos crônicos.

\begin{tabular}{|c|c|c|c|c|c|c|}
\hline \multirow[b]{2}{*}{ Xenodiagnóstico } & \multirow[b]{2}{*}{ Exame/dias } & \multicolumn{5}{|c|}{$\mathrm{N}^{\circ}$ de triatomíneos } \\
\hline & & aplicados & examinados & \multicolumn{2}{|c|}{ positivos } & $\begin{array}{c}\text { mortos } \\
\%\end{array}$ \\
\hline \multirow[t]{2}{*}{ Artificial } & $1 \%$ & 2.280 & 2.186 & 14 & 0,64 & 4,3 \\
\hline & $3 \%$ & & 2.095 & 36 & 1,71 & 2,5 \\
\hline \multirow[t]{2}{*}{ Natural } & $1 \% / 30$ & 2.280 & 2.236 & 10 & 0,44 & 2,0 \\
\hline & $3 \%$ & & 2.113 & 21 & 0,99 & 3,5 \\
\hline
\end{tabular}

Tabela 3 - Estudo comparativo do nünero médio de Trypanosoma cruzi excretados por lâmina (laminnila $22 x 22$ mun) de triatomineos aplicados 110 xenodiagnóstico natural e artifictal, em pacientes chagásicos na fase crônica. utilizando o método das dejeçōes espontâneas.

\begin{tabular}{cccr} 
Leitura/ & & \multicolumn{2}{c}{ Xenodiagnóstico } \\
\cline { 2 - 4 } dias & Pacientes & Artificial & Natural \\
\hline $1^{\circ} / 30$ & 20 & $31,7 \pm 20,22$ & $3,1 \pm 1.51$ \\
$2^{\circ} / 60$ & 20 & $44,8 \pm 24,32$ & $15,8 \pm 7.57$ \\
$3^{\circ} / 90$ & 20 & $78,2 \pm 43,57$ & $41,6 \pm 19,32$ \\
\hline
\end{tabular}

látex" '. Neste trabalho, utilizou-se o preservativo masculino não lubrificado, devido aos excelentes resultados já obtidos ${ }^{12} 22$. Além disso, tem sido utilizado durante vários anos, na rotina do nosso laboratório, para criação de triatomíneos com alimentação artificial. Para que não haja mortalidade desses insetos, é fundamental que se atenda ao cuidado de não manuseá-los logo após a alimentação, porque quaisquer traumatismos lhes são fatais. Outros fatores favoráveis, que contribuíram na decisão do tipo de membrana, foram: a disponibilidade no comércio, a facilidade de conservação e a praticidade no uso.

A opção pela heparina teve como base o estudo comparado de anticoagulantes em relação à replicação do $T$. $c r u z l^{512}$.
O primeiro trabalho ${ }^{16}$ a mostrar a possibilidade do uso do xenodiagnóstico artificial, após a descrição do método natural', foi através da utilização de tubos de ensaio com sangue citratado ou desfibrinado de pessoas suspeitas de serem chagásicas. Esses tubos eram cobertos com pele de cobaio recém-sacrificado, mantidos em contato com os triatomíneos em estufa a $37^{\circ} \mathrm{C}$ e esses foram examinados entre um e dois meses depois, demonstrando com esse método a "xenopositividade" em alguns casos, sem, no entanto, citarem dados quantitativos consistentes. Posteriormente, um estudo ${ }^{15}$, comparando a sensibilidade do xenodiagnóstico natural e o artificial, foi realizado em cobaios inoculados com o $T$. cruzi que apresentavam parasitos detectáveis 
Santos AH, Silva IG, Rassi A. Estudo comparativo entre o xenodiagnóstico natural e o artificial em chagásicos crônicos. Revista da Sociedade Brasileira de Medicina Tropical 28:367-373, out-dez, 1995.

em exame a fresco. Neste trabalho, os autores detectaram o tripanosoma em cinco dos oito triatomíneos aplicados no xenodiagnóstico natural e em sete dos dezesseis triatomíneos usados no xenodiagnóstico artificial. O estudo comparativo dos xenodiagnósticos natural e artificial foi realizado a partir da aplicação simultânea dos dois métodos, em 57 pacientes chagásicos na fase crônica, com idade superior a 26 anos. Os resultados mostraram que o método artificial foi mais eficiente para a comprovação parasitológica da doença de Chagas do que o natural. A sensibilidade de uma aplicação do xenodiagnóstico artificial correspondeu a três aplicações do método natural. Desta forma, sugere-se o uso do método artificial em rotina de laboratório, não só em substituição ao natural, como também para evitar as reações cutâneas de hipersensibilidade, de anafilaxia, devendo ser recomendado para paciente imunodeprimido, transplantado ou alérgico à saliva do triatomíneo. Além disso, oferece mais uma opção de exame que é, psicologicamente, mais aceitável pelo paciente.

Foi demonstrado ${ }^{6}$ em 210 pacientes e, em 76 pacientes chagásicos na fase crônica ${ }^{22}$, que a faixa etária de 10 a 20 anos foi a de maior positividade do xenodiagnóstico, decrescendo nas faixas etárias subseqüentes e elevando-se novamente nas faixas superiores a 50 anos. Isto é concordante com os dados deste trabalho. A faixa etária de 41 a 55 anos foi a mais difícil para a detecção do T. cruzi. Não foram encontrados dados na literatura que explicassem este fato. No entanto, a hipótese mais provável é a em que o paciente, nesta faixa etária, apresenta maior resposta imunológica, diminuindo desta forma a parasitemia circulante. Neste trabalho, em ambos os métodos, "xenopositividade" foi significativamente maior no sexo masculino, nas faixas etárias entre 56-65 anos e 66-70 anos.

De acordo com vários autores ${ }^{6}{ }^{13} 22$, as idades compreendidas entre 30 e 49 anos têm sido as de menor positividade. Neste trabalho, 20 dos 57 pacientes estão dentro desta faixa etária e isto talvez tenha influenciado na positividade final de $35,1 \%$, que foi inferior à encontrada na literatura ${ }^{22}$ que usando a mesma metodologia, encontrou uma positividade de $55,3 \%$, e outro trabalho com três repetições ${ }^{6}$, uma positividade de 69,3\%. Contudo, essa positividade de $35,1 \%$ é concordante com as encontradas por outros autores ${ }^{18} 19$.

A repetição do xenodiagnóstico é um fator favorável na detecção de tripanosoma ${ }^{2} 817$. Porém, existem variáveis intrínsecas ao paciente no qual a repetição não evidencia o tripanosoma ${ }^{18}$, como apresentado em 12 séries mensais aplicadas em 66 chagásicos crônicos. No entanto, um trabalho ${ }^{6}$ mostra a positividade do xenodiagnóstico de $41,2 \%$ na primeira aplicação e que aumentou em $19,5 \%$ e $8,6 \%$, respectivamente, na segunda e terceira aplicações, dando uma positividade final de $69,3 \%$. Neste trabalho, a xenopositividade de $21,1 \%$, elevou-se em $2,3 \%$ e $6,4 \%$, respectivamente, no segundo e terceiro exames, dando uma positividade acumulada de $29,8 \%$.

Quanto às leituras aos 30, 60 e 90 dias, os resultados deste trabalho evidenciaram que não há necessidade do exame aos 90 dias, uma vez que a positividade do xenodiagnóstico foi a mesma aos 60 dias, nos dois métodos e nas repetições, usando o método das dejeções espontâneas.

A mortalidade dos triatomíneos submetidos ao xenodiagnóstico artificial foi de 4,3\%, 1,8\% e $2,5 \%$, respectivamente, no $1^{\circ}, 2^{\circ}$ e $3^{\circ}$ exames, e no método natural de $2,0 \%, 2,2 \%$ e $3,5 \%$. Verificou-se, que quando se usou o método das dejeções espontâneas, a mortalidade foi cerca de três vezes menor, do que a verificada utilizando o método da compressão abdominal ${ }^{20}$.

\section{SUMMARY}

In order to study the sensitivity of the xenodiagnosis technique a comparison between natural and artificial xenodiagnosis methods was performed in 57 chronic phase chagasic patients (31 female), with ages ranging from 26 to 83 years. All patients bad demonstrable antibodies against Trypanosoma cruzi. Forty first instar nymphs of Dipetalogaster maximus were used for each of both methods and for each patient. The positivity of xenodiagnosis artificial was significantly bigher than the routine test method. These results did show that a single application of 40 bugs by the artificial method yielded a similar result than 3 applications of 40 bugs each, by the natural metbod.The positivity of xenodiagnosis was significantly bigher in patients between 56-65 and 66-83 years old than at other ages. Males were predominant in this age 
group. These results showed the viability of artificial xenodiagnosis and its use in routine laboratory testing

Key-words: Xenodiagnosis. Chagas' disease. Trypanosoma cruzi. Chronic phase. Parasitological diagnosis.

\section{REFERÊNCIAS BIBLIOGRÁFICAS}

1. Balazuc J. Un fenomeno de anafilaxia producido por picaduras de Triatoma (Hemiptera, Reduviidae). Anales del Instituto de Medicina Regional 3:35-37, 1950.

2. Boainain E, Rassi A. Quadro clínico e diagnóstico. Publicação da Roche 4:22-24, 1976.

3. Bronfen E, Rocha FSA, Machado GBN, Perillo MM, Romanha AJ, Chiari E. Isolamento de amostras do Trypanosoma cruzi por xenodiagnóstico $\mathrm{e}$ hemocultura de pacientes na fase crônica da doença de Chagas. Memórias do Instituto Oswaldo Cruz 84:237-240, 1989.

4. Campos R, Amato Neto V, Matsubara L, Moreira AAB, Pinto PLS. Estudos sobre o xenodiagnóstico "in vitro". I. Escolha de anticoagulante e de membranas. Revista do Hospital da Clínicas da Faculdade de Medicina de São Paulo 43:101-103, 1988.

5. Castro CN, Alves MT, Macedo VO. Importância da repetição do xenodiagnóstico para avaliação da parasitemia na fase crônica da doença de Chagas. Revista da Sociedade Brasileira de Medicina Tropical 16:98-103, 1983.

6. Cedillos RA, Torrealba JW, Tonn RJ, Mosca W, Ortegon A. El xenodiagnóstico artificial en la enfermedad de Chagas. Boletin de la Oficina Sanitaria Panamericana 93:240-249, 1982.

7. Cerisola JA, Rohdwedder R. Comportamiento de la parasitemia y el inmunodiagnóstico de la infección chagásica crónica. In: Resumos do Simposio Internacional sobre Enfermedad de Chagas. Buenos Aires p.271-275, 1972.

8. Chiari E. Padronização do xenodiagnóstico. Revista da Sociedade Brasileira de Medicina Tropical 25(supl III):40-42, 1992.

9. Freitas JLP, Nussensweig V, Amato Neto V, Sontag R. Estudo comparativo entre xenodiagnóstico

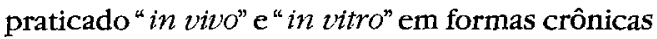
da moléstia de Chagas. O Hospital 47:181-187, 1955.

10. Garcia ES, Macarini JD, Garcia MLM, Ubatuba FB. Alimentação de Rhodnius prolixus em laboratório. Anais da Academia Brasileira de Ciências 47:537-545, 1975.

11. Isac E. Influência da heparina e do citrato de sódio no xenodiagnóstico artificial. Revista de Patologia Tropical 23:121-143, 1994.

12. Luquetti AO, Miles MA, Rassi A, Rezende JM, Souza AA, Póvoa MM, Rodrigues I. Trypanosoma cruzi: zymodemes associated with acute and chronic Chagas' disease in central Brazil. Transactions of the Royal Society of Tropical Medicine and Hygiene 80:462-470, 1986.

13. Marsden PD, Barreto AC, Cuba CC, Gama MB, Akers J. Improvements in routine xenodiagnosis with first instar Dipetalogaster maximus (Uhler, 1894) (Triatominae). The American Journal of Tropical Medicine and Hygiene 28:649-652, 1979.

14. Nussensweig V, Sonntag R. Xenodiagnóstico artificial. Novo processo. Primeiros resultados positivos. Revista Paulista de Medicina 40:69-71, 1952.

15. Romaña C, Gil J. Xenodiagnóstico artificial. Anales del Instituto de Medicina Regional 2:57-60, 1947.

16. Salgado AA. Consideraciones sobre metodología y sensibilidad del xenodiagnóstico. Boletin Chileno de Parasitologia 24:9-13, 1969.

17. Salgado AA, Mayrink W, Dias JCP. Estudo comparativo entre a reação de fixação do complemento, com os antígenos benzeno-cloroformado e metílico e o xenodiagnóstico. Revista do Instituto de Medicina Tropical de São Paulo 12:36-40,1970.

18. Salgado, JA, Salgado AA, Espinola HN. Contribuição ao conhecimento das reações às picadas de triatomíneos. Revista Brasileira de Malariologia e Doenças Tropicais 20:231-235, 1968.

19. Schenone H, Rojo M, Rojas A, Concha L. Positividad diurna y nocturna del xenodiagnostico en un paciente con infección chagásica crónica de parasitemia permanente. Boletin Chileno de Parasitologia 32:63-66, 1977.

20. Silva IG. Influência da temperatura na biologia de triatomíneos. I. Triatoma rubrovaria (Blanchard, 1843) (Hemiptera, Reduviidae). Revista Goiana de Medicina 31:1-37, 1985.

21. Silva IG. Influência da temperatura na biologia de 18 espécies de triatomíneos (Hemiptera, Reduviidae) e no xenodiagnóstico. Tese de Doutorado, Universidade Federal do Paraná, Curitiba, PR, 1985. 
Santos $A H$, Silva IG, Rassi A. Estudo comparativo entre o xenodiagnóstico natural e o artificial em chagásicos crônicos. Revista da Sociedade Brasileira de Medicina Tropical 28:367-373, out-dez, 1995.

22. Silva IG. Influência da temperatura na biologia de triatomíneos. XIII. Dipetalogaster maximus Uhler, 1894 (Hemiptera, Reduviidae). Anais da Sociedade Entomológica do Brasil 19:111-119, 1990.

23. Silva IG. Nova técnica para leitura do xenodiagnóstico. Revista Goiana de Medicina 36:35-40, 1990.

24. Silva IG. Dispositivo para realização do xenodiagnóstico artificial. Revista de Patologia Tropical 20:35-38, 1991.

25. Silva IG, Ferreira IR. Influência da fonte sangüínea na multiplicação da cepa " $Y$ " de Trypanosoma cruzi em Triatoma infestans (Klug, 1834) e Rhodnius neglectus Lent, 1954. Revista Goiana de Medicina 36:41-48, 1990.

26. Silva IG, Luquetti AO, Silva HHG. Importância do método de obtenção das dejeções espontâneas dos triatomíneos na avaliação da suscetibilidade triatomínica para o Trypanosoma cruzi. Revista da Sociedade Brasileira de Medicina Tropical 26: 19-24, 1993.

27. Silva IG, Silva HHG. Influência da temperatura na biologia de triatomíneos. IX. Rhodnius nasutus Stal, 1859 (Hemiptera, Reduviidae). Memórias do Instituto Oswaldo Cruz 84:377-382, 1989.

28. Silva IG, Silva HHG. Suscetibilidade de 11 espécies de triatomíneos (Hemiptera, Reduviidae) à cepa Y de Trypanosoma cruzi (Kinetoplastida, Trypanosomatidae). Revista Brasileira de Entomologia 37:459-463, 1993.

29. Silva $\Pi$. Sobre la conveniencia de realizar el xenodiagnóstico fuera del organismo humano en todos los casos. Revista de la Facultad Medicina de Tucumán 1:405-415, 1958. 